\title{
Spectral properties of the one-dimensional two-channel Kondo lattice model
}

\author{
A. M. Tsvelik \\ Department of Physics, University of Oxford, 1 Keble Road, Oxford, OX1 3NP, U.K. \\ C. I. Ventura * \\ Centro Atómico Bariloche, 8400-Bariloche, Argentina.
}

\begin{abstract}
We have studied the energy spectrum of a one-dimensional Kondo lattice, where the localized magnetic moments have $\mathrm{SU}(\mathrm{N})$ symmetry and two channels of conduction electrons are present. At half filling, the system is shown to exist in two phases: one dominated by RKKY-exchange interaction effects, and the other by Kondo screening. A quantum phase transition point separates these two regimes at temperature $T=0$. The Kondo-dominated phase is shown to possess soft modes, with spectral gaps much smaller than the Kondo temperature.
\end{abstract}

PACS numbers: 71.27.+a,71.10,75.30.Mb

In this paper we discuss a one-dimensional two-channel $\mathrm{SU}(\mathrm{N})$-symmetric Kondo lattice, that is, a 1-D lattice where spin operators are represented by generators of the $\operatorname{su}(\mathrm{N})$ algebra and the conduction band has an additional two-fold degeneracy. We discuss the case where each conduction band is half-filled. At this point due to the additional particle-hole symmetry the total symmetry of the model is $\mathrm{SU}(\mathrm{N}) \times \mathrm{Z}_{2} \times \mathrm{SU}(2)$.

The interest in this model is justified by the fact that the corresponding impurity model exhibits non-Fermi liquid (NFL) behaviourd, which als takes place for the $D=\infty$ two-channel Kondo lattices. The origin of this NFL behaviour in the single impurity problem is quite clear - it is caused by dispersionless modes (see Refs. [3] ) .

Our study of the half-filled lattice case demonstrates that the system can exist in two phases: the RKKY dominated phase, where its low-energy behaviour is determined by the RKKYl exchange interactions between local magnetic moments induced by the conduction electrons, and the Kondo-dominated or heavy fermion state, where Kondo screening of the magnetic impurities takes place. Both phases are separated by a quantum phase transition point at $T=0$. This agrees with the scenario for the Kondo lattice suggested by Doniach $\mathbf{3}$. The heavy fermion phase possesses soft modes; these modes have spectral gaps which are much smaller than the Kondo temperature $\left(T_{K}\right)$.

Most of the papers devoted to the Kondo lattice use the slave-boson approach and the large- $\mathrm{N}$ approximation $\mathrm{G}$, which is more suitable for the Kondo dominated (disordered) phase. The opposite situation is easier to study in one dimension, where the RKKY interaction has a simpler form. This has been done by one of the authors for the one-dimensional M-channel Kondo lattice at halffillingl: when the spin sector has $\mathrm{SU}(2)$ symmetry. In this case both charge and orbital sectors acquire gaps, while for half-integer value of $|M / 2-S|$ the spin sector remains gapless and at low energies is described by the
$\mathrm{SU}_{1}(2)$ Wess-Zumino-Novikov-Witten model (WZNW) In this paper we generalize this analysis for $\mathrm{SU}(\mathrm{N})$ symmetry of the spin sector: the existence of an additional parameter, N, opens a possibility to study a competition between the RKKY- and the Kondo-dominated regimes.

We consider the following Hamiltonian:

$$
\begin{aligned}
H= & -t \sum_{i ; m=1, N ; \alpha=1,2} c_{i, m, \alpha}^{+} c_{i+1, m, \alpha} \\
& +J \sum_{i ; m, l=1, N ; \alpha=1,2} c_{i, m, \alpha}^{+} d_{i, m} d_{i, l}^{+} c_{i, l, \alpha}+H . c .
\end{aligned}
$$

Here $i$ is the lattice site index, $m(=1, \ldots N)$ numbers components of the $\mathrm{SU}(\mathrm{N})$ spin, while $\alpha$ is the channel index of the conduction electrons. $c_{i, m, \alpha}^{+}$is the creation operator for conduction electrons in a state characterized by quantum numbers $i, m, \alpha$, while $d_{i, m}^{+}$corresponds to the local moment states. The Hamiltonian is supplemented by the constraint

$$
\sum_{m=1, N} d_{i, m}^{+} d_{i, m}=q
$$

where the number $q$ represents the filling of the local orbitals. It remains finite in the limit $N \longrightarrow \infty$.

The calculations for the RKKY regime are similar to the ones for the $\mathrm{SU}(2)$-symmetric case done int. The mean field gap in the charge sector is $\Delta \sim J$, while in the Kondo regime it is $T_{K} \sim D e^{\frac{-2 \pi}{N J \rho\left(\epsilon_{F}\right)}}$, being $D$ the conduction band bandwidth and $\rho\left(\epsilon_{F}\right) \sim D^{-1}$ the density of states at the Fermi level. For large $N$, the latter gap is the largest one.

The appearance of the gap leads to the change of the ground state energy; in the RKKY-phase we have

$$
E_{R K K Y}=-\frac{N \rho\left(\epsilon_{F}\right) \Delta^{2}}{\pi} \ln \frac{D}{\Delta} \approx-\frac{N \rho\left(\epsilon_{F}\right) J^{2}}{\pi} \ln \frac{D}{J}
$$

and in the Kondo regime it is: 


$$
-\frac{N T_{K}}{4 \pi}=\frac{N}{4 \pi} e^{\frac{-2 \pi}{N \rho\left(\epsilon_{F}\right)}}
$$

The critical value of $N$ at which both ground state energies become comparable is colossal for any realistic $J$ :

$$
N_{c} \sim-\frac{\pi}{J \rho\left(\epsilon_{F}\right)} \ln ^{-1}\left[1 / J \rho\left(\epsilon_{F}\right)\right]
$$

but it does not matter since we are going to study universal properties of the spectrum.

Let us now discuss the properties of the system on both sides of the transition. Deep in the RKKY phase the adequate description of the spin sector in the continuum limit is given by the $\frac{U(N)}{U(N / 2) \otimes U(N / 2)}$ non-linear sigma model $(\mathrm{NL} \sigma)$ with the topological term $\mathrm{g}$. In the limit $N=2$ this becomes the $S U(2) / U(1)$ model with the topological term obtained for this case in Ref. []. In the presence of the topological term the model is likely to be massless. The transmutation scale (that is the energy scale at which the model undergoes a crossover to the strong coupling regime) of this model is:

$$
\Delta_{s p} \sim J e^{\frac{-2 \pi}{N g}}, \quad g \sim \rho\left(\epsilon_{F}\right) J \sqrt{\ln (D / J)} .
$$

On the other hand, in the Kondo limit the spin sector most certainly has a gap (we shall see that it is much smaller than $T_{K}$ ). Since the NL $\sigma$ model is critical, its renormalization group beta function 10 has a zero at some finite value of $g=\rho\left(\epsilon_{F}\right) J N$ (see Fig.1). If there is no 1st order phase transition between the two regimes, the beta function can be continued into the Kondo regime where the spectrum has a gap. For such behaviour to take place, one needs to have a beta function which does not cross the horizontal axis but just touches it.

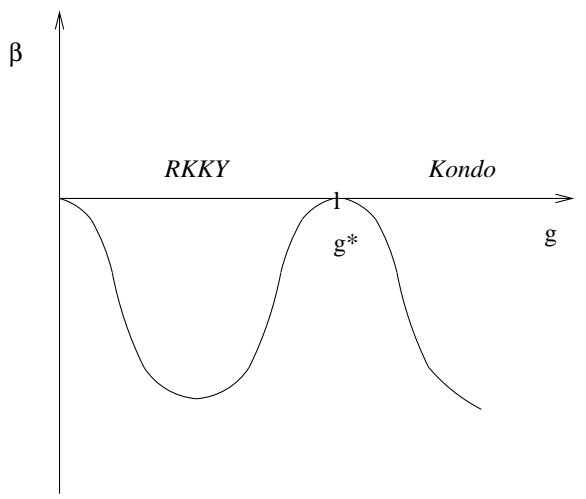

Fig. 1. Schematic plot of the beta function as a function of the bare coupling.

This behaviour of the $\beta$ function corresponds to the presence of a marginal operator at the critical point.

We will now discuss the Kondo-dominated (large $N$ ) regime in detail. The Lagrangian density can be written as follows:

$$
\begin{aligned}
L= & \sum_{i, j ; m=1, N ; \alpha=1,2} c_{i, m, \alpha}^{+}\left[\partial_{\tau} \delta_{i, j}-\hat{\epsilon}_{i, j}\right] c_{j, m, \alpha} \\
& +\sum_{i ; m=1, N} d_{i, m}^{+} \partial_{\tau} d_{i, m} \\
& +\frac{J}{N} \sum_{i ; m, l=1, N ; \alpha=1,2} c_{i, m, \alpha}^{+} d_{i, m} d_{i, l}^{+} c_{i, l, \alpha} \\
& +i \lambda \sum_{i ; m=1, N}\left(d_{i, m}^{+} d_{i, m}-q\right)+\text { H.c. }
\end{aligned}
$$

In this expression $\lambda$ is a Lagrange multiplier introduced to enforce the local constraint on occupation number of the localized orbitals.

We next decouple the interaction term employing the Hubbard-Stratonovich transformation:

$$
\begin{array}{r}
\frac{J}{N} \sum_{i ; m, l=1, N ; \alpha=1,2} c_{i, m, \alpha}^{+} d_{i, m} d_{i, l}^{+} c_{i, l, \alpha} \longrightarrow \\
\frac{N V_{i, \alpha}^{+} V_{i, \alpha}}{2 J}+\sum_{i ; m=1, N ; \alpha=1,2}\left[V_{i, \alpha} c_{i, m, \alpha}^{+} d_{i, m}+H . c .\right],
\end{array}
$$

where $V_{i, \alpha}=|V| z_{i, \alpha}$ and $\sum_{\alpha=1,2} z_{i, \alpha}^{*} z_{i, \alpha}=1$. We now apply the following further transformation for the conduction band orbitals:

$$
c_{i, m, \alpha}=z_{i, \alpha} \chi_{i, m}+\epsilon_{\alpha, \beta} z_{i, \beta}^{*} \eta_{i, m},
$$

which can be cast also in the following matricial form:

$$
\left(\begin{array}{c}
c_{i, m, 1} \\
c_{i, m, 2}
\end{array}\right)=\left(\begin{array}{cc}
z_{i, 1} & z_{i, 2}^{*} \\
z_{i, 2} & -z_{i, 1}^{*}
\end{array}\right)\left(\begin{array}{c}
\chi_{i, m} \\
\eta_{i, m}
\end{array}\right)=-\hat{g}_{i}\left(\begin{array}{c}
\chi_{i, m} \\
\eta_{i, m}
\end{array}\right)
$$

Here $\hat{g}$ is a matrix belonging to $\mathrm{SU}(2)$.

One can imagine two simple-minded mean field configurations now:

a) the uniform one, in which the $\hat{g}$ matrices on different sites are equal;

b) the case $z_{2 i+1, \alpha}=\epsilon_{\alpha, \beta} z_{2 i, \beta}^{*}$, which in matrix form would read:

$$
\hat{g}_{2 i+1}=-\hat{g}_{2 i}\left(\begin{array}{cc}
0 & -1 \\
1 & 0
\end{array}\right) .
$$

In the first case, in mean field approximation only one conduction band channel ( $\chi$-chain) hybridizes with the local orbitals. Meanwhile, in case (b) both chains are similarly hybridized (see Fig.2).

The spectrum in case (b) is determined by the following eigenvalue equation:

$$
\omega\left(w^{2}-4 \cos ^{2}(k / 2) V^{2}\right)=0,
$$

so that there is a solution $\omega=0$. The appearance of such solution indicates that this configuration is energetically unstable. This conclusion is supported by the following calculations. 


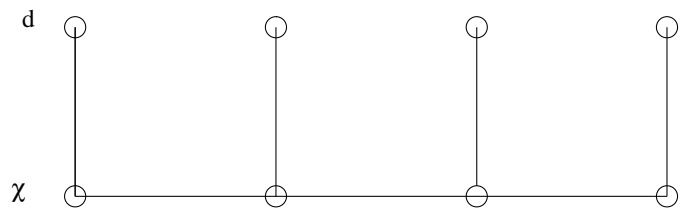

$\eta$

(a)

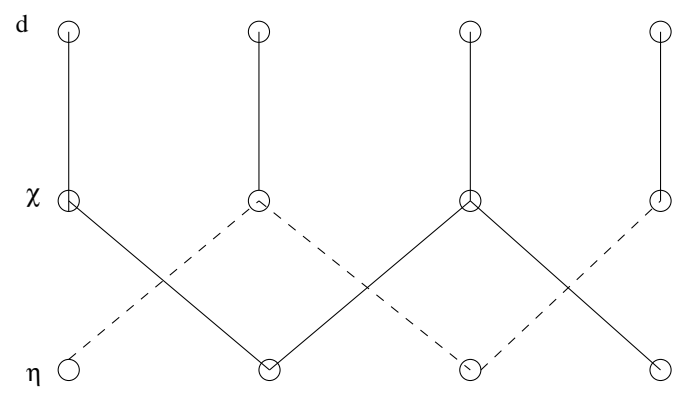

( b )

Fig. 2. Pictorial view of the hybridization of the chains. Cases (a) and (b) as described in text.

The self-consistency equation determining $V$ is:

$$
V=N J<\chi_{i, m} d_{i, m}^{+}>,
$$

which, upon introducing the one-dimensional tightbinding dispersion relation for the conduction bands, yields the following expressions for $V$, respectively, in the two cases:

$$
\begin{aligned}
& 1=\frac{N J}{2 \pi} \int_{-\pi / 2}^{\pi / 2} \frac{d k}{\sqrt{\sin ^{2} k+V_{(a)}^{2}}}, \\
& 1=\frac{N J}{2 \pi} \int_{0}^{\pi / 2} \frac{d k}{\sqrt{\sin ^{2} k+\frac{V_{(b)}^{2}}{4}}} .
\end{aligned}
$$

One can see that $V_{(a)}$ for the uniform mean field solution is larger than $V_{(b)}$ ( by a factor of $e^{\frac{\pi}{N J}}$ ), thus the ground state energy is likely to be lower in the uniform solution as we now proceed to show.

The ground state energy is determined by the derivative of the free energy with respect to the parameter $V$ :

$$
\frac{\partial F}{\partial V}=\frac{V}{J}+\frac{N}{2}\left(<\chi_{i, m} d_{i, m}^{+}>+<d_{i, m} \chi_{i, m}^{+}>\right),
$$

which in the uniform case leads to:

$$
F=\frac{1}{2}\left(\frac{V_{(a)}^{2}}{J}-\frac{N V_{(a)}^{2}}{2 \pi} \ln \frac{e}{V_{(a)}^{2}}\right)
$$

At the minimum this leads to $F_{0}^{(a)}=-\frac{N V_{(a)}^{2}}{4 \pi}$. Similarly, one gets for the other mean field solution: $F_{0}^{(b)}=-\frac{N V_{(b)}^{2}}{8 \pi}$, which has higher energy than the uniform mean field solution.

Let us now consider fluctuations around the uniform mean-field solution. We write the $\hat{g}$ matrix in terms of two slow fields, as follows:

$$
\hat{g}_{n}=G(x)\left(\sqrt{1-\vec{l}^{2}(x)}+i(-1)^{n} \vec{\sigma} \cdot \vec{l}(x)\right),
$$

where $|\vec{l}(x)|<<1$ and $G(x)$ belongs to $\mathrm{SU}(2)$.

Neglecting fluctuations in $V$ we get the following action on the lattice:

$$
\begin{aligned}
S= & \Psi_{n}^{+}\left(\partial_{\tau}+G^{+} \partial_{\tau} G\right) \Psi_{n}+\Psi_{n}^{+} \hat{\epsilon}\left[i \partial_{x}+a \partial_{x} G^{+} G\right] \Psi_{n} \\
& +V\left(\chi_{n, m}^{+} d_{n, m}+c . c\right)+d_{n, m}^{+} \partial_{\tau} d_{n, m} \\
& +i \lambda(x)\left(d_{n, m}^{+} d_{n, m}-\frac{N}{2}\right) \Psi_{n+1}^{+}\left(-2 l^{2}\right) \Psi_{n} \\
& +i(-1)^{n} \Psi_{n}^{+} \vec{\sigma} \cdot \vec{l}(x) \Psi_{n} ; \quad \Psi_{n}=\left(\begin{array}{c}
\chi_{n, m} \\
\eta_{n, m}
\end{array}\right) .
\end{aligned}
$$

Let us now decompose the unhybridized fermion into slowly varying components,

$$
\eta_{n, m}=R(x)(i)^{n}+L(x)(-i)^{n},
$$

where $x=n a$ ( $a$ is the lattice parameter), substitute this into the above effective action and integrate over fast fermionic fields $\chi, d$ with energies above the gap: $\sim$ $\frac{V^{2}}{D}=T_{K}$, and the field $l$. After the integration we get the following effective action for the flavour and the spin sectors:

$$
\begin{aligned}
S & =S[P C F]+S[G N], \\
S[P C F] & =\frac{N}{2 \pi} \operatorname{Tr}\left(\partial_{\tau} G^{+} \partial_{\tau} G\right)+\frac{N}{2 \pi} v^{2} \operatorname{Tr}\left(\partial_{x} G^{+} \partial_{x} G\right), \\
S[G N] & =\bar{\psi}_{m} \gamma_{\mu} \partial_{\mu} \psi_{m}-\frac{\pi}{32 N}\left(\bar{\psi}_{m} \psi_{m}\right)^{2},
\end{aligned}
$$

where $v \sim v_{F}$ and the spinor $\psi$ incorporates the $R, L$ fields. Both effective actions have $T_{K}$ as their ultraviolet cut-off.

The action for $G$ must vanish in the limit $V \longrightarrow 0$. This will happen through the shrinking of the cutoff: $T_{K} \longrightarrow 0$.

The action in Eq.(21) corresponds to the Principal Chiral Field model on the SU(2) group. It is exactly solvable11; the excitations have a spectral gap

$$
\Delta_{G} \sim T_{K} e^{-N}
$$

Fermionic model (22), describing the stin sector, is the $\mathrm{O}(2 \mathrm{~N})$-symmetric Gross-Neveu mode 12 with the bare coupling constant $g=\pi / 16 N$. It is also exactly solvable and all excitations have gaps 13 . The mass scale is 


$$
\Delta_{\psi} \sim T_{K} e^{-\frac{\pi}{g(N-2)}} \sim T_{K} e^{-16}
$$

This gap is numerically small despite the fact that it does not contain $N$.

Thus, we see that the heavy fermion phase contains two modes, the spectra of which lie well inside of the singleparticle gap. One mode is magnetic ( the one described by the Gross-Neveu model) and the other is not.

The present work was supported by a Joint Collaboration grant awarded by the British Council and Fundación Antorchas. The authors acknowledge the hospitality of their institutions throughout their respective stays abroad.

* Member of the Carrera del Investigador Científico of CONICET (Consejo Nacional de Investigaciones Científicas y Técnicas, Argentina).

${ }^{1}$ A. M. Tsvelik and P. B. Wiegmann, Z. für Phys. B54, 201 (1984); N. Andrei and C. Destri, Phys. Rev. Lett. 52, 364 (1984); A. M. Tsvelik, J. Phys. C17, 2299 (1984); A. W. W. Ludwig and I. Affleck, Phys. Rev. Lett. 57, 3160(1991).

${ }^{2}$ M. Jarrell et al., Phys. Rev. Lett. 77, 1612(1996); F. B. Anders, M. Jarrell and D. L. Cox, Phys. Rev. Lett. 78, 2000(1997).

${ }^{3}$ V. Emery and S. Kivelson, Phys. Rev. B47, 10812 (1992); P. Coleman, L. Ioffe and A. M. Tsvelik, Phys. Rev. B52, 6611 (1995).

${ }^{4}$ M. A. Ruderman and C. Kittel, Phys. Rev. 96, 99 (1954).

${ }^{5}$ S. Doniach, Physica B 91, 231 (1977).

${ }^{6}$ P. Coleman, Phys. Rev. B35, 5072(1987).

${ }^{7}$ A. M. Tsvelik, Phys. Rev. Lett. 72, 1048(1994).

${ }^{8}$ S. Novikov, Usp. Math. Nauk. 37, 3 (1982); E. Witten, Comm. Math. Phys. 92, 455 (1984); A. M. Polyakov and P. B. Wiegmann, Phys. Lett. B141, 223 (1984).

${ }^{9}$ N. Read and S. Sachdev, Nucl. Phys. B316, 609 (1989).

${ }^{10}$ M. Gell-Mann and F. E. Low, Phys. Rev. 95, 1300 (1954); see also e.g. "Quantum Field Theory and Critical Phenomena", by J. Zinn-Justin, Oxford University Press (1993).

${ }^{11}$ A. M. Polyakov and P. B. Wiegmann, Phys. Lett. B131, $121(1983)$

${ }^{12}$ D. J. Gross and A. Neveu, Phys. Rev. D10, 3235 (1974).

${ }^{13}$ A. B. Zamolodchikov and Al. B. Zamolodchikov, Ann. Phys. (N.Y.) 120, 253 (1979); Nucl. Phys. B133, 525 (1978); P. Forgács, F. Niedermayer and P. Weisz, Nucl. Phys. B367, 123 (1991). 